\title{
Co-occurrence of diabetes and hopelessness predicts adverse prognosis following percutaneous coronary intervention
}

\author{
Susanne S. Pedersen · Johan Denollet • \\ Ruud A. M. Erdman · Patrick W. Serruys • \\ Ron T. van Domburg
}

Accepted: January 22, 2009/Published online: February 7, 2009

(c) The Author(s) 2009. This article is published with open access at Springerlink.com

\begin{abstract}
We examined the impact of co-occurring diabetes and hopelessness on 3-year prognosis in percutaneous coronary intervention patients. Consecutive patients $(n=534)$ treated with the paclitaxel-eluting stent completed a set of questionnaires at baseline and were followed up for 3-year adverse clinical events. The incidence of 3 -year death/non-fatal myocardial infarction was 3.5\% in patients with no risk factors (neither hopelessness nor diabetes), $8.2 \%$ in patients with diabetes, $11.2 \%$ in patients with high hopelessness, and $15.9 \%$ in patients with both factors $(p=0.001)$. Patients with hopelessness (HR: 3.28; 95\% CI: 1.49-7.23) and co-occurring diabetes and hopelessness (HR: 4.89 ; 95\% CI: 1.86-12.85) were at increased risk of 3-year adverse clinical events compared to patients with no risk factors, whereas patients with diabetes were at a clinically relevant but not statistically significant risk (HR: 2.40; 95\% CI: 0.82-7.01). These results remained, adjusting for baseline characteristics and depressive symptoms. These findings testify to the importance of identifying patients with co-occurring risk factors, as they
\end{abstract}

S. S. Pedersen $(\bowtie) \cdot$ J. Denollet

Department of Medical Psychology, CoRPS-Center

of Research on Psychology in Somatic diseases,

Tilburg University, Room P506, Warandelaan 2, PO Box 90153,

5000 LE Tilburg, The Netherlands

e-mail: s.s.pedersen@uvt.nl

S. S. Pedersen - R. A. M. Erdman · P. W. Serruys ·

R. T. van Domburg

Department of Cardiology, Thoraxcenter, Erasmus Medical

Center, Rotterdam, The Netherlands

R. A. M. Erdman

Department of Medical Psychology and Psychotherapy,

Erasmus Medical Center, Rotterdam, The Netherlands likely require special management in clinical practice in addition to standard medical treatment.

Keywords Depressive symptoms - Diabetes ·

Hopelessness - Percutaneous coronary intervention .

Prognosis

\section{Introduction}

Patients with co-morbid diabetes and coronary artery disease (CAD) have a higher risk for adverse prognosis and restenosis following successful percutaneous coronary intervention (PCI), due to manifestations of CAD being more severe in patients with diabetes (The Bari Investigators 1997; Detre et al. 1999; Buse et al. 2007). Psychosocial factors, such as depression and hopelessness, may enhance this risk. In CAD, depression has been associated with multiple adverse health outcomes, including increased mortality (Barth et al. 2004; de Jonge et al. 2006; Jiang et al. 2001), re-hospitalizations (Jiang et al. 2001), poor health status (Rumsfeld et al. 2003; Ruo et al. 2003), and non-compliance (Dimatteo et al. 2000). Similarly, in patients with diabetes, depression contributes to increased morbidity and mortality (Ciechanowski et al. 2000), decreased quality of life (Hanninen et al. 1999), and poor glycemic control (Gary et al. 2000).

Studies have examined the separate influence of diabetes and depression on clinical outcome in CAD rather than their co-occurrence or adjusted statistically for one factor while examining the impact of the other. However, co-occurring diabetes and depression likely incur a higher risk of adverse clinical events than the presence of one of the factors (Rozanski et al. 2005). In a recent study of PCI patients, we found that diabetes predicted the onset of 
depressive symptoms at 12 months in patients free of symptoms at 6 months (Pedersen et al. 2006), suggesting that patients with co-occurring diabetes and CAD may comprise high-risk patients. In the recent enhancing recovery in coronary heart disease patients (ENRICHD) trial on patients with increased psychosocial risk (i.e., being either depressed or having low perceived social support, or both), both diabetes and depression were independent predictors of death or non-fatal myocardial infarction (MI), but the study did not examine whether depression modulates the impact of diabetes on outcome (Jaffe et al. 2006). By 2015, 30\% of all revascularization procedures in the US are estimated to be undertaken in patients with diabetes (Kapur et al. 2005). Hence, knowledge of a potential combined adverse effect of diabetes and depression on prognosis in patients treated with PCI is important for secondary prevention.

In the studying of the co-occurrence of diabetes and depression on prognosis, we have chosen to focus on hopelessness rather than depression per se. Although hopelessness may be considered a feature of depression, it is neither a necessary nor a sufficient criterion for a clinical diagnosis of depression according to DSM-IV criteria. In addition, it correlates weakly with standard depression scales, and therefore may deserve studying in its own right (Everson et al. 1996). Others have also found that hopelessness is associated with reduced survival in a mixed group of CAD patients (Barefoot et al. 2000). In addition, the studying of hopelessness fits in well with the ongoing quest for the identification of the nature of depressive symptoms and those that have the most adverse effect on prognosis (de Jonge et al. 2006). Hence, the aim of the current prospective 3 -year follow-up study was to examine the impact of co-occurring diabetes and hopelessness on prognosis in PCI patients, adjusting for a standardized measure of depressive symptoms.

\section{Materials and methods}

Patients and design

Consecutive patients with stable or unstable angina, undergoing PCI with the paclitaxel-eluting stent as the default strategy (i.e., $88.8 \%$ of the current sample was treated with the paclitaxel-eluting stent) at the Erasmus Medical Center, Rotterdam, The Netherlands, between July 1, 2003 and July 1, 2004, comprised the patient sample for the current study. The study design has been described elsewhere (Pedersen et al. 2007). In brief, 710 patients were approached in writing 4 weeks post-PCI (referred to as baseline in the remainder of the article) and asked to complete a set of standardized and validated questionnaires, of whom $536 \quad(75 \%$ response rate) agreed. Responders and non-responders differed on smoking and dyslipidemia, with non-responders being more likely to smoke $(22 \%$ vs. $14 \% ; p=0.003)$ but less likely to suffer from dyslipidemia $(63 \%$ vs. $74 \% ; p=0.001)$ than responders. No other differences were found between nonresponders and responders on baseline characteristics, including cardiac medication. Since two patients had not responded to the item assessing hopelessness in the current study, statistical analyses are based on 534 patients.

The hospital medical ethics committee approved the study protocol. The study was carried out conform to the Helsinki Declaration.

\section{Measures}

\section{Demographic and clinical characteristics}

Demographic variables comprised sex, age, and marital status. Information on clinical variables, including indication for PCI (stable vs. unstable angina), stent type (paclitaxel-eluting stent vs. other), multi-vessel disease, cardiac history [MI, coronary artery bypass graft surgery (CABG) or PCI prior to the index PCI], hypertension, dyslipidemia, diabetes mellitus, smoking, and cardiac medication (aspirin, beta-blockers, ACE-inhibitors, diuretics, and statins) was obtained from the medical records. Hypertension, dyslipidemia and diabetes mellitus were considered present if patients were treated for the condition.

\section{Hopelessness}

Hopelessness was assessed with the item "Have you experienced a feeling of hopelessness recently?" of the Maastricht Questionnaire at baseline (Appels et al. 1987). The item is answered on a 3 -point scale $(0=$ no; $1=$ ?; 2 = yes). This item has been shown to be of prognostic value in PCI patients, with patients responding with a 1 or 2 to the item being defined as suffering from hopelessness (Pedersen et al. 2007). Previous studies have also used 1 or 2 items to assess hopelessness as a risk factor for the onset of CAD (Everson et al. 1996; Pollitt et al. 2005; Everson et al. 1997) and prognosis in CAD (Pedersen et al. 2007).

\section{Depressive symptoms}

We used the depression subscale of the Hospital Anxiety and Depression Scale (HADS) to evaluate the presence of depressive symptoms at baseline (Zigmond and Snaith 1983; Spinhoven et al. 1997). The subscale consists of 7 items that are answered on a 4-point Likert scale from 0 to 
3 (score range $0-21$ ). A cut-off $\geq 8$ on the subscale was used to indicate patients with likely depressive symptomatology, as this represents an optimal balance between sensitivity and specificity (Bjelland et al. 2002). The subscale is a valid and internally consistent measure of depressive symptoms, with Cronbach's alphas ranging from 0.67 to 0.90 across 15 studies (Bjelland et al. 2002). An advantage of using the HADS in the context of CAD is that the measure is largely devoid of somatic items, decreasing the chance that scores are confounded by cardiac disease severity (Herrmann 1997).

\section{Clinical outcome}

Clinical outcome was defined as a cumulative endpoint of either death (all-cause) or non-fatal MI 3 years post-PCI. If patients would experience more than one event during the follow-up period, the first occurring event would count. The municipal civil registry was contacted to ascertain the survival status of every patient. Information on non-fatal MIs was obtained from the medical records, using an increase in the creatine kinase-MB fraction of 3 times the upper limit of normal as the defining criteria, according to guidelines of the American Heart Association and the American College of Cardiology (Smith et al. 2006; Smith et al. 2001).

\section{Statistical analysis}

The correlation between hopelessness and depressive symptoms at baseline was examined with Spearman's rho $(\rho)$, since the scale related to hopelessness was at an ordinal level. To ascertain the impact of co-occurring versus single risk factors, four groups were formed on the basis of the risk factors diabetes and hopelessness, as follows: (1) No risk factors (neither diabetes nor hopelessness); (2) Diabetes only; (3) Hopelessness only; (4) Cooccurring diabetes and hopelessness. Baseline characteristics stratified by diabetes and hopelessness were compared with the Chi-square test (Fisher's exact test when appropriate) for nominal variables and analysis of variance (ANOVA) with a post-hoc Bonferroni correction for continuous variables. Univariable and multivariable Cox regression analyses were used to determine the impact of the four risk groups on clinical outcome at 3 years, using the no risk group as the reference category. A priori, the covariates sex, age, multi-vessel disease, cardiac history (MI, PCI or CABG prior to the index PCI), hypertension, dyslipidemia, and smoking were chosen for inclusion in the multivariable analyses based on the literature. Baseline variables on which the four risk groups differed were also entered as covariates, as they may potentially serve as confounders on the impact of the four risk groups on clinical events. In addition, we entered depressive symptoms, using a cut-off $\geq 8$ on the HADS at baseline, to ascertain that hopelessness exerted an independent effect of depressive symptoms on clinical outcome. Hazard ratios (HR) with their associated $95 \%$ confidence intervals (CI) are reported. A $p$-value $<0.05$ was used to indicate statistical significance. All tests were two-tailed. All analyses were performed using SPSS 14.0 for Windows (SPSS Inc., Chicago, Illinois).

\section{Results}

Relationship between hopelessness and depressive symptoms

The correlation between hopelessness and depressive symptoms at baseline was $\rho=0.43(p<0.001)$, indicating a shared variance of $18 \%$. There was no indication that the correlation between hopelessness and depressive symptoms was larger in patients with multi-vessel disease $(n=321$; $\rho=0.41 ; p<0.001)$ compared to patients with no multivessel disease $(n=213 ; \rho=0.47 ; p<0.001)$.

Number of events at 3-year follow-up

In total 38 patients experienced either a non-fatal MI or died during the 3-year follow-up period, with one patient experiencing both events, with the non-fatal MI preceding death in this patient. For statistical analyses, the first event, that is the non-fatal MI, counted towards the endpoint.

\section{Baseline characteristics}

Baseline characteristics stratified by diabetes and hopelessness are shown in Table 1. Differences were found between risk factor groups on age, multi-vessel disease, hypertension, dyslipidemia, current smoking, and the use of ACE-inhibitors. Patients with diabetes were older, more likely to have hypertension and dyslipidemia than the other three groups, whereas high hopelessness patients were more likely to smoke and use ACE-inhibitors. Patients with co-occurring diabetes and hopelessness were more likely to have multi-vessel disease. No other differences on demographic and clinical baseline characteristics were found between groups.

The prevalence of depressive symptoms at baseline, using the standardized cut-off $\geq 8$ on HADS [25], was $13.0 \%$ in patients with neither risk factors, $16.4 \%$ in patients with diabetes, $48.6 \%$ in the high hopelessness 
Table 1 Patient characteristics stratified by hopelessness and diabetes*

\begin{tabular}{|c|c|c|c|c|c|}
\hline & None $(n=286)$ & Diabetes $(n=61)$ & Hopelessness $(n=143)$ & Co-occurrence $(n=44)$ & $p$ \\
\hline \multicolumn{6}{|l|}{ Demographics } \\
\hline Males & 217 (75.9) & $40(65.6)$ & $98(68.5)$ & $30(68.2)$ & 0.21 \\
\hline Age, mean (SD) & $63.7(10.6)$ & $67.4(10.4)$ & $60.3(11.5)$ & 64.2 & $<0.001$ \\
\hline Single/no partner & $51(18.0)$ & $15(25.0)$ & $27(19.0)$ & $9(20.9)$ & 0.65 \\
\hline \multicolumn{6}{|l|}{ Indication for PCI } \\
\hline Unstable angina & $137(47.9)$ & $25(41.0)$ & $63(44.1)$ & $21(47.7)$ & 0.73 \\
\hline \multicolumn{6}{|l|}{ Stent type } \\
\hline Paclitaxel-eluting stent ${ }^{\mathrm{a}}$ & $254(88.8)$ & $52(85.2)$ & $132(92.3)$ & $37(84.1)$ & 0.31 \\
\hline \multicolumn{6}{|l|}{ Clinical variables } \\
\hline Multi-vessel disease & $167(58.4)$ & $42(68.9)$ & $76(53.1)$ & $36(81.8)$ & 0.003 \\
\hline Cardiac history $^{\mathrm{b}}$ & $145(50.7)$ & $35(57.4)$ & $87(60.8)$ & $28(63.6)$ & 0.14 \\
\hline Hypertension & $124(43.4)$ & $48(78.7)$ & $54(37.8)$ & $30(68.2)$ & $<0.001$ \\
\hline Dyslipidemia & $202(70.6)$ & $56(91.8)$ & $98(68.5)$ & $39(88.6)$ & $<0.001$ \\
\hline Current smoking & $36(12.6)$ & $5(8.2)$ & $30(21.0)$ & $4(9.1)$ & 0.032 \\
\hline \multicolumn{6}{|l|}{ Cardiac medication } \\
\hline Aspirin & $276(96.5)$ & $58(95.1)$ & $132(92.3)$ & $42(95.5)$ & 0.29 \\
\hline Beta-blockers & $58(20.3)$ & $13(21.3)$ & $37(25.9)$ & $10(22.7)$ & 0.62 \\
\hline ACE-inhibitors & $13(4.5)$ & $6(9.8)$ & $23(16.1)$ & 7 (15.9) & $<0.001$ \\
\hline Diuretics & $4(1.4)$ & $1(1.6)$ & $3(2.1)$ & $1(2.3)$ & 0.86 \\
\hline Statins & $211(73.8)$ & $43(70.5)$ & 109 (76.2) & $35(79.5)$ & 0.72 \\
\hline
\end{tabular}

* Values are expressed as $n(\%)$ unless otherwise specified

a The paclitaxel-eluting stent (PES) was used as the default strategy (i.e., in $88.8 \%$ of the total sample); other stents used were the sirolimuseluting stent (SES; 4\%), both PES and SES (1\%), or bare metal stent/balloon dilation (6\%)

${ }^{\mathrm{b}} \mathrm{MI}, \mathrm{PCI}$ or CABG prior to the index PCI

patients, and $50 \%$ in patients with co-occurring diabetes and hopelessness $(p<0.001$; Fig. 1). Hence, we added depressive symptoms at baseline as a covariate to the multivariable Cox regression analysis.

Incidence of clinical events 3-year post-PCI stratified by diabetes and hopelessness

The incidence of death or non-fatal MI at follow-up differed significantly between the four risk groups, with $3.5 \%$ in patients with no risk factors (neither diabetes nor hopelessness), $8.2 \%$ in patients with diabetes, $11.2 \%$ in the high hopelessness patients, and $15.9 \%$ in patients with co-occurring hopelessness and diabetes ( $p=0.001$; Fig. 2). In univariable analysis using the no risk factor group as the reference category, patients with hopelessness (HR: 3.28; 95\% CI: $1.49-7.23 ; p=0.003)$ and co-occurring diabetes and hopelessness (HR: 4.89; 95\% CI: $1.86-12.85 ; p=$ 0.001 ) were at increased risk of 3 -year adverse clinical events compared to patients with no risk factors, whereas patients with diabetes had a clinically relevant risk although it was not statistically significant (HR: 2.40 ; $95 \%$ CI: $0.82-$ $7.01 ; p=0.11)$.

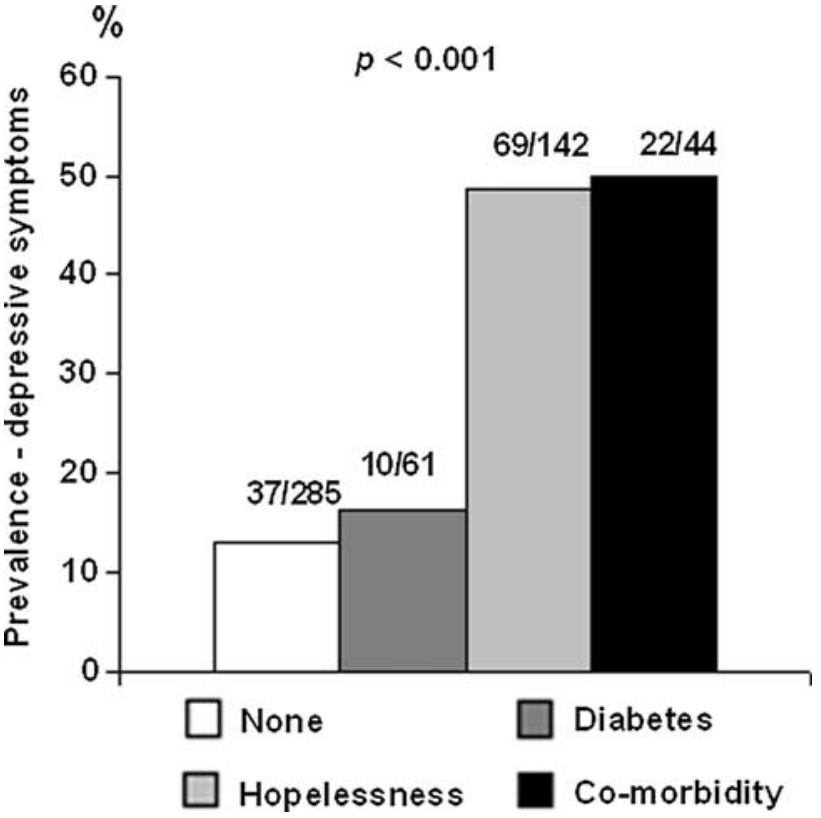

Fig. 1 Prevalence of depressive symptoms (cut-off $\geq 8$ ) at baseline stratified by diabetes and hopelessness (total numbers are listed on top of bars; two patients had no score on the HADS, hence this analysis is based on $n=532$ ) 


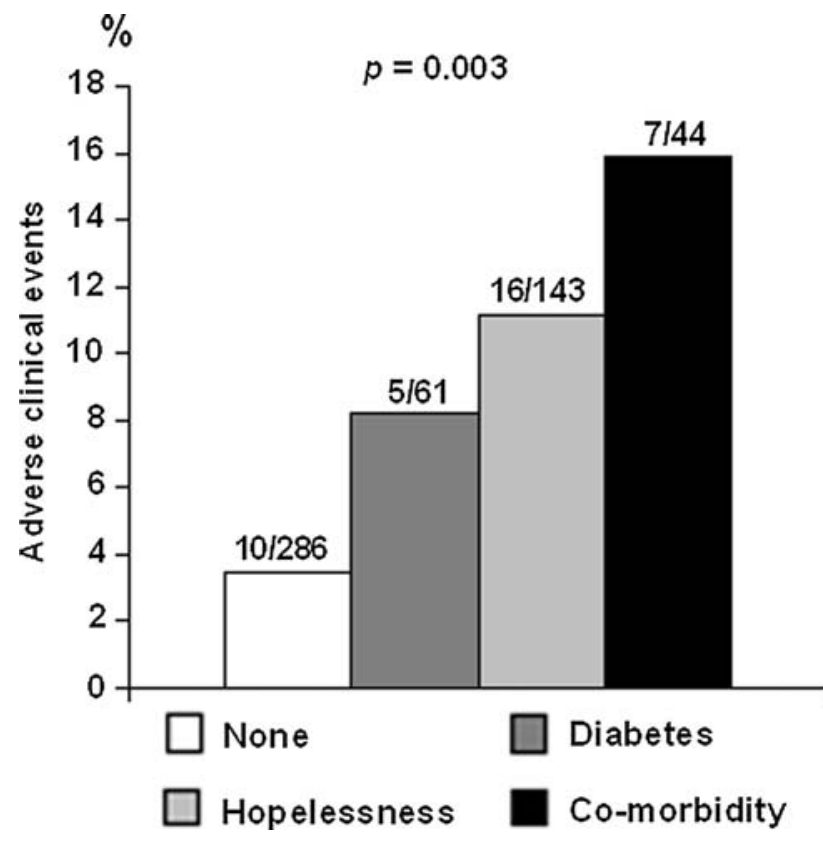

Fig. 2 Risk of death or non-fatal myocardial infarction 3 years postPCI (total numbers are listed on top of bars)

Impact of diabetes and hopelessness on 3-year clinical events (adjusted analysis)

Results of the impact of co-occurring versus single risk factors are shown in Table 2. Patients with co-occurring diabetes and hopelessness (HR: 5.12; 95\% CI: 1.79-14.64; $p=0.002$ ) remained at the highest risk for adverse prog-

Table 2 Impact of diabetes and hopelessness on 3-year clinical events (adjusted analysis)

\begin{tabular}{llll}
\hline & HR & {$[95 \% \mathrm{CI}]$} & $p$ \\
\hline Diabetes* $^{*}$ & 2.28 & {$[0.75-6.96]$} & 0.15 \\
Hopelessness* & 3.45 & {$[1.48-8.01]$} & 0.004 \\
$\begin{array}{l}\text { Co-occurring diabetes } \\
\quad \text { and hopelessness* }\end{array}$ & 5.12 & {$[1.79-14.64]$} & 0.002 \\
Depressive symptoms (baseline) & 0.68 & {$[0.32-1.45]$} & 0.32 \\
Male sex & 1.02 & {$[0.48-2.16]$} & 0.95 \\
Age & 1.02 & {$[0.99-1.06]$} & 0.15 \\
Multi-vessel disease & 1.81 & {$[0.78-4.16]$} & 0.17 \\
Cardiac history & 1.92 & {$[0.89-4.14]$} & 0.10 \\
Hypertension & 0.95 & {$[0.47-1.91]$} & 0.88 \\
Dyslipidemia & 0.59 & {$[0.28-1.23]$} & 0.16 \\
Smoking & 0.91 & {$[0.30-2.77]$} & 0.86 \\
ACE-inhibitors & 1.53 & {$[0.65-3.56]$} & 0.33 \\
\hline
\end{tabular}

* No risk group (neither diabetes nor hopelessness) $=$ reference category nosis at 3 years, followed by high hopelessness patients (HR: 3.45 ; 95\% CI: $1.48-8.01 ; p=0.004$ ), adjusting for demographic (sex and age) and clinical factors (multivessel disease, CAD history, hypertension, dyslipidemia, smoking, and ACE-inhibitors) and depressive symptoms at baseline. Diabetes as a single risk factor again fell short of significance (HR: $2.28 ; 95 \%$ CI: $0.75-6.96 ; p=0.15$ ). Depressive symptoms (HR: 0.68; 95\% CI: 0.32-1.45; $p=0.32$ ) were not a significant predictor of prognosis, but there was a trend for CAD history (HR: 1.92; 95\% CI: $0.89-4.14 ; p=0.096$ ).

In order to further examine the relationship found for the impact of single risk factors (i.e., diabetes or hopelessness) versus co-occurring diabetes and hopelessness, we used the Wald statistics to test the statistical significance of each coefficient. All differences between the risk factor groups were significant $(p s<0.05)$.

\section{Discussion}

In the current study, we examined the impact of co-occurring diabetes and hopelessness on prognosis in patients with established CAD in general and PCI patients treated with drug-eluting stents in particular. The 3-year incidence of death/MI was highest in patients with co-occurring risk factors $(15.9 \%)$, followed by the high hopelessness patients $(11.2 \%)$, patients with diabetes $(8.2 \%)$, and patients with no risk factors (i.e., neither diabetes nor hopelessness; $3.5 \%$ ). Patients with co-occurring diabetes and hopelessness were at a 5-fold risk of death or non-fatal MI at 3 years, with the risk to patients with a single risk factor (i.e., diabetes or hopelessness) being 2- to 3 -fold compared to patients with no risk factors, controlling for demographic and clinical risk factors and depressive symptoms at baseline.

Psychosocial risk factors are known to cluster together within individuals and also to act in synergy with traditional risk factors for CAD (Rozanski et al. 2005). In addition, mood disorders, such as depression, have been shown to exacerbate the burden of physical illness (Fenton and Stover 2006) and to lead to more extensive health-care use in the physically ill (Stein et al. 2006), with co-morbid depression and diabetes also increasing the risk of mortality compared to patients with depression or diabetes alone (Egede et al. 2005). Similar to a population-based study of 10,025 individuals showing that co-occurring diabetes and depression was associated with a greater risk for cardiac- and all-cause mortality compared with the single risk factors alone (Egede et al. 2005), we found that co-occurring diabetes and hopelessness incurred the highest risk of a composite of mortality and non-fatal MI 3 years post-PCI. 
It is noteworthy that hopelessness and co-occurring diabetes and hopelessness had an impact on prognosis above and beyond depressive symptoms at baseline. However, this finding is consistent with the results of others, showing that hopelessness predicts mortality in CAD patients (Pedersen et al. 2007) and the incidence of cancer, MI, and mortality in the general population independent of depression (Everson et al. 1996). In addition, others have shown that hopelessness correlates weakly with depression (Everson et al. 1996), which was also confirmed in the current study with an overlap in variance of $18 \%$. These results testify to the prognostic properties of hopelessness and emphasize the importance of studying hopelessness in its own right (Everson et al. 1996; Breitbart et al. 2000). Further research into hopelessness and its potential mechanisms also fits in well with the ongoing quest for the identification of the depressive symptoms that have the most adverse effect on prognosis (de Jonge et al. 2006).

In clinical practice, identifying patients who score high on hopelessness can easily be done with the 1-item measure of the MQ used in the current study. Alternatively, the 2-item patient health questionnaire (PHQ-2; Löve et al. 2005) and the 4-item Symptoms of mixed AnxietyDepression index $\left(\mathrm{SAD}_{4}\right.$; Denollet et al. 2006) both comprise an item assessing hopelessness. The former measure has been shown to be sensitive to change and the latter to predict major depressive disorder, adjusting for depressive symptoms. From a treatment point of view, enhancing partner support or patients' perception of this support may increase a sense of optimism and reduce hopelessness (Gustavsson-Lilius et al. 2007). Cognitive behavioral therapy may well form the key to changing patients' perception, although it may be necessary to use it in combination with anti-depressant treatment in more severe cases. Although conducted in college students, a recent randomized controlled trial using a brief mailed intervention for depressive symptoms as the mainstay of treatment also offers hope to patients characterized by feelings of hopelessness (Geisner et al. 2006). The latter study showed that a reduction in depressive symptoms was partly mediated by a reduction in hopelessness, emphasizing the importance of the therapist instilling hope of change in patients. Future intervention studies should see if current interventions impact on hopelessness as a way to improve clinical outcomes and quality of life.

This study has some limitations. First, $25 \%$ of patients declined to participate, and non-responders differed on smoking and dyslipidemia, although not on any of the other demographic and clinical baseline characteristics. Second, the sample consisted of patients with stable or unstable angina as indication for PCI, and the results may not generalize to patients with acute MI. Nevertheless, psychosocial factors have been shown to predict mortality in these patients, emphasizing the relevance of also studying the impact of co-occurring risk factors in MI patients (Lespérance et al. 2000). Our results support this notion. Third, we cannot rule out the possibility that the impact of diabetes on prognosis may be due to lack of adherence, which we did not assess. Adherence to cardio-protective medication in patients with co-morbid diabetes and CAD has been linked to prognosis (Ho et al. 2006). We also had no information on health-related behaviors, such as physical activity and dietary intake, which could mediate the relationship between hopelessness and clinical events. Fourth, we had no information on the use of psychotropic medication, such as anti-depressants, and participation in cardiac rehabilitation, which could potentially serve as confounders. Fifth, the number of events was relatively small with 38 deaths and non-fatal MIs, which means that essentially the multivariable model was overfitted. It is possible that given more events, diabetes as a single risk factor would also have been significantly associated with prognosis in the current study. Sixth, the measures were obtained 4 weeks post-procedure, with the possibility that a healthy survivor effect could exist. However, logistically it was not possible to assess patients closer to the index event. In addition, there is no consensus as to the most optimal time point for assessing psychological distress, including depression, in cardiac patients (Nicholson et al. 2006). In fact, a recent study of depression in PCI patients showed that baseline assessment was a less strong predictor of subsequent depression compared to assessment at 1 month (Poston et al. 2003).

In conclusion, co-occurring diabetes and hopelessness was associated with a 5-fold increased risk of 3-year prognosis post-PCI, controlling for baseline demographic and clinical risk factors and depressive symptoms. The risk incurred by the co-occurrence of these two risk factors was higher than that for the single risk factors (i.e., diabetes and hopelessness). These findings testify to the importance of identifying patients with co-occurring risk factors, as they likely require special management in clinical practice including additional intervention, e.g., of a behavioral nature, in addition to pharmacological treatment. It now seems timely to shift our focus from studying the impact of single risk factors on prognosis to their co-occurrence, focusing on risk factors of both a clinical and psychosocial nature.

Acknowledgments This research was supported by the Netherlands Organization for Scientific Research (NWO) with a VENI grant (451-05-001) to Dr. S. S. Pedersen and a VICI grant (453-04-004) to Dr. J. Denollet.

Open Access This article is distributed under the terms of the Creative Commons Attribution Noncommercial License which permits any noncommercial use, distribution, and reproduction in any medium, provided the original author(s) and source are credited. 


\section{References}

Appels, A., Höppener, P., \& Mulder, P. (1987). A questionnaire to assess premonitory symptoms of myocardial infarction. International Journal of Cardiology, 17, 15-24. doi:10.1016/01675273(87)90029-5.

Barefoot, J. C., Brummett, B. H., Helms, M. J., et al. (2000). Depressive symptoms and survival of patients with coronary artery disease. Psychosomatic Medicine, 62, 790-795.

Barth, J., Schumacher, M., \& Herrmann-Lingen, C. (2004). Depression as a risk factor for mortality in patients with coronary heart disease: A meta-analysis. Psychosomatic Medicine, 66, 802-813. doi:10.1097/01.psy.0000146332.53619.b2.

Bjelland, I., Dahl, A. A., Haug, T. T., et al. (2002). The validity of the hospital anxiety and depression scale: An updated literature review. Journal of Psychosomatic Research, 52, 69-77. doi: 10.1016/S0022-3999(01)00296-3.

Breitbart, W., Rossenfeld, B., Pessin, H., et al. (2000). Depression, hopelessness, and desire for hastened death in terminally ill patients with cancer. Journal of the American Medical Association, 284, 2907-2911. doi:10.1001/jama.284.22.2907.

Buse, J. B., Ginsberg, H. N., Bakris, G. L., et al. (2007). Primary prevention of cardiovascular diseases in people with diabetes mellitus: A scientific statement from the American Heart Association and the American Diabetes Association. Circulation, 115, 114-126. doi:10.1161/CIRCULATIONAHA.106.179294.

Ciechanowski, P. S., Katon, W. J., \& Russo, J. E. (2000). Depression and diabetes: Impact of depressive symptoms on adherence, function, and costs. Archives of Internal Medicine, 160, 32783285. doi:10.1001/archinte.160.21.3278.

de Jonge, P., Ormel, J., van den Brink, R. H. S., et al. (2006). Symptom dimensions of depression following myocardial infarction and their relationship with somatic health status and cardiovascular prognosis. The American Journal of Psychiatry, 163, 138-144. doi:10.1176/appi.ajp.163.1.138.

Denollet, J., Strik, J. J., Lousberg, R., et al. (2006). Recognizing increased risk of depressive comorbidity after myocardial infarction: Looking for 4 symptoms of anxiety-depression. Psychotherapy and Psychosomatics, 75, 346-352. doi:10.1159/ 000095440.

Detre, K. M., Guo, P., Holubkov, R., et al. (1999). Coronary revascularization in diabetic patients: A comparison of the randomised and observational components of the bypass angioplasty revascularization investigation (BARI). Circulation, 99, 633-640.

Dimatteo, M. R., Lepper, H. S., \& Croghan, T. W. (2000). Depression is a risk factor for noncompliance with medical treatment: Metaanalysis of the effects of anxiety and depression in patient adherence. Archives of Internal Medicine, 160, 2101-2107. doi: 10.1001/archinte.160.14.2101.

Egede, L., Niertert, P., \& Zheng, D. (2005). Depression and all-cause and coronary heart disease mortality among adults with and without diabetes. Diabetes Care, 28, 1339-1345. doi:10.2337/ diacare.28.6.1339.

Everson, S. A., Goldberg, D. E., Kaplan, G. A., et al. (1996). Hopelessness and risk of mortality and incidence of myocardial infarction and cancer. Psychosomatic Medicine, 58, 113121.

Everson, S. A., Kaplan, G. A., Goldberg, D. E., et al. (1997). Hopelessness and 4-year progression of carotid atherosclerosis: The Kupio ischemic heart disease risk factor study. Arteriosclerosis, Thrombosis, and Vascular Biology, 17, 1490-1495.

Fenton, W. S., \& Stover, E. S. (2006). Mood disorders: Cardiovascular and diabetes comorbidity. Current Opinion in Psychiatry, 19, 421-427. doi:10.1097/01.yco.0000228765.33356.9f.
Gary, T. L., Crum, R. M., Cooper-Patrick, L., et al. (2000). Depressive symptoms and metabolic control in African-Americans with type 2 diabetes. Diabetes Care, 23, 23-29. doi: 10.2337/diacare.23.1.23.

Geisner, I. M., Neighbors, C., \& Larimer, M. E. (2006). A randomized clinical trial of a brief, mailed intervention for symptoms of depression. Journal of Consulting and Clinical Psychology, 74, 393-399. doi:10.1037/0022-006X.74.2.393.

Gustavsson-Lilius, M., Julkunen, J., \& Hietanen, P. (2007). Quality of life in cancer patients: The role of optimism, hopelessness, and partner support. Quality of Life Research, 16, 75-87. doi: 10.1007/s11136-006-9101-4.

Hanninen, J. A., Takala, J. K., \& Keinanen-Kiukaanniemi, S. M. (1999). Depression in subjects with type 2 diabetes: Predictive factors and relation to quality of life. Diabetes Care, 22, 997998. doi:10.2337/diacare.22.6.997.

Herrmann, C. (1997). International experiences with the hospital anxiety and depression scale: A review of validation data and clinical results. Journal of Psychosomatic Research, 42, 17-42. doi:10.1016/S0022-3999(96)00216-4.

Ho, P. M., Magid, D. J., Masoudi, F. A., et al. (2006). Adherence to cardioprotective medications and mortality among patients with diabetes and ischemic heart disease. BMC Cardiovascular Disorders, 6, 48. doi:10.1186/1471-2261-6-48.

Jaffe, A. S., Krumholz, H. M., Catellier, D. J., Enhancing Recovery in Coronary Heart Disease Patients (ENRICHD) Trial Investigators, et al. (2006). Prediction of medical morbidity and mortality after acute myocardial infarction patients at increased psychosocial risk in the enhancing recovery in coronary heart disease patients (ENRICHD) study. American Heart Journal, 152, 26-35. doi:10.1016/j.ahj.2005.10.004.

Jiang, W., Alexander, J., Christopher, E., et al. (2001). Relationship of depression to increased risk of mortality and rehospitalization in patients with congestive heart failure. Archives of Internal Medicine, 161, 1849-1856. doi:10.1001/archinte.161.15.1849.

Kapur, A., Malik, I. S., Bagger, J. P., et al. (2005). The coronary artery revascularisation in diabetes (CARDia) trial: Background, aims, and design. American Heart Journal, 149, 13-19. doi: 10.1016/j.ahj.2004.07.001.

Lespérance, F., Frasure-Smith, N., Juneau, M., et al. (2000). Depression and 1-year prognosis in unstable angina. Archives of Internal Medicine, 160, 1354-1360. doi:10.1001/archinte.160. 9.1354 .

Löve, B., Kroenke, K., \& Gräfe, K. (2005). Detecting and monitoring depression with a two-item questionnaire (PHQ-2). Journal of Psychosomatic Research, 58, 163-171. doi:10.1016/j.jpsychores. 2004.09.006.

Nicholson, A., Kuper, H., \& Hemingway, H. (2006). Depression as an aetiologic and prognostic factor in coronary heart disease: A meta-analysis of 6362 events among 146538 participants in 54 observational studies. European Heart Journal, 27, 2763-2774. doi:10.1093/eurheartj/ehl338.

Pedersen, S. S., Ong, A. T. L., Serruys, P. W., et al. (2006). Type D Personality and diabetes predict the onset of depressive symptoms in patients following percutaneous coronary intervention. American Heart Journal, 151, 367.e1-367.e6.

Pedersen, S. S., Denollet, J., Daemen, J., et al. (2007). Fatigue, depressive symptoms, and hopelessness as predictors of clinical events following percutaneous coronary intervention with paclitaxel-eluting stents. Journal of Psychosomatic Research, 62, 455-461. doi:10.1016/j.jpsychores.2006.12.018.

Pollitt, R. A., Daniel, M., Kaufman, J. S., et al. (2005). Mediation and modification of the association between hopelessness, hostility, and progression of carotid atheroslcerosis. Journal of Behavioral Medicine, 28, 53-64. doi:10.1007/s10865-005-2563-y. 
Poston, W. S. C., Haddock, C. K., Conard, M. W., et al. (2003). Assessing depression in the cardiac patient. When is the appropriate time to assess depression in the patient undergoing coronary revascularization? Behavior Modification, 27, 26-36. doi:10.1177/0145445502238691.

Rozanski, A., Blumenthal, J. A., Davidson, K. W., et al. (2005). The epidemiology, pathophysiology, and management of psychosocial risk factors in cardiac practice: the emerging field of behavioral cardiology. Journal of the American College of Cardiology, 45, 637-651. doi:10.1016/j.jacc.2004.12.005.

Rumsfeld, J. S., Havranek, E., Masoudi, F. A., Cardiovascular Outcomes Research Consortium, et al. (2003). Depressive symptoms are the strongest predictors of short-term declines in health status in patients with heart failure. Journal of the American College of Cardiology, 42, 1811-1817. doi:10.1016/ j.jacc.2003.07.013.

Ruo, B., Rumsfeld, J. S., Hlatky, M. A., et al. (2003). Depressive symptoms and health-related quality of life: The heart and soul study. Journal of the American Medical Association, 290, 215221. doi:10.1001/jama.290.2.215.

Smith, S. C., Dove, J. T., Jacobs, A. K., et al. (2001). ACC/AHA guidelines for percutaneous coronary intervention (revision of the 1993 PTCA guidelines) - executive summary: A report of the american college of cardiology/american heart association task force on practice guidelines (Committee to revise the 1993 guidelines for percutaneous transluminal coronary angioplasty) endorsed by the Society for cardiac angiography and interven- tions. Journal of the American College of Cardiology, 37, 22152238.

Smith, S. C., Feldman, T. E., Hirshfeld, J. W., et al. (2006). ACC/ AHA/SCAI 2005 Guideline update for percutaneous coronary intervention-summary article: A report of the american college of cardiology/american heart association task force on practice guidelines (ACC/AHA/SCAI writing committee to Update the 2001 guidelines for percutaneous coronary intervention). Journal of the American College of Cardiology, 47, 216-235. doi: 10.1016/j.jacc.2005.11.025.

Spinhoven, P., Ormel, J., Sloekers, P. P., et al. (1997). A validation study of the hospital anxiety and depression scale (HADS) in different groups of Dutch subjects. Psychological Medicine, 27, 363-370. doi:10.1017/S0033291796004382.

Stein, M. B., Cox, B. J., Afifi, T. O., et al. (2006). Does co-morbid depressive illness magnify the impact of chronic physical illness? Psychological Medicine, 36, 587-596. doi:10.1017/S00 33291706007239.

The BARI Investigators. (1997). Influence of diabetes on 5-year mortality and morbidity in a randomized trial comparing CABG and PTCA in patients with multivessel disease: The bypass angioplasty revascularization investigation (BARI). Circulation, 96, 1761-1769.

Zigmond, A. S., \& Snaith, R. P. (1983). The hospital anxiety and depression scale. Acta Psychiatrica Scandinavica, 67, 361-370. doi:10.1111/j.1600-0447.1983.tb09716.x. 